\title{
Management of healthcare areas for the prevention of COVID-19 emergency in an Italian teaching hospital in Pisa, Tuscany: A hospital renovation plan
}

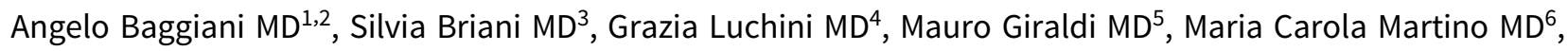 \\ Andrea Porretta $M D^{1,2}$, Michele Totaro $\mathrm{MD}^{2}$ and Gaetano Privitera $\mathrm{MD}^{1,2}$ \\ ${ }^{1}$ Hygiene and Epidemiology Unit, Azienda Ospedaliero Universitaria Pisana, Pisa, Italy, ${ }^{2}$ Department of Translational Research and the New Technologies in \\ Medicine and Surgery, University of Pisa, Pisa, Italy, ${ }^{3}$ General Management Unit, Azienda Ospedaliero Universitaria Pisana, Pisa, Italy, ${ }^{4} \mathrm{Health}$ Management Unit, \\ Azienda Ospedaliero Universitaria Pisana, Pisa, Italy, ${ }^{5} \mathrm{Hospital}$ Medical Direction Unit, Azienda Ospedaliero Universitaria Pisana, Pisa, Italy and ${ }^{6}$ Disaster Manager \\ Unit, Azienda Ospedaliero Universitaria Pisana, Pisa, Italy
}

To the Editor-The current outbreak of coronavirus disease 2019 (COVID-19), which began in Wuhan, China, in December 2019, has spread to $>160$ countries in $<3$ months. In March 2020, the World Health Organization (WHO) defined this outbreak as a pandemic emergency that requires strategies to manage the infectious risk, including social distancing measures and border closures. ${ }^{1,2}$

In Italy, a cluster of cases began in Lombardy on February 21, and by March 1, the virus had spread to all Italian regions. From February 21 to March 24, a total of 69,176 cases were confirmed, with 6,820 deaths. The Tuscany region has registered 2,699 cases with 918 hospitalized patients. Of these 918 patients, 244 (27\%) had respiratory failure and required specialized care in an intensive care unit (ICU). ${ }^{3}$ Considering the limited number of beds in ICUs, we describe the technical emergency measures adopted by a 1,081-bed teaching hospital located in the Pisa district (Tuscany, Italy) that aimed to prevent hospital overcrowding by COVID19 patients.

Following the first confirmed COVID-19 case in Tuscany on February 25, a task force assembled with representatives from several hospital areas: medical direction, hygiene and epidemiology, facility management, occupational medicine, emergency medicine, and intensive care units. After drafting a procedure for COVID-19 patient management, the working group divided the hospital and emergency department into COVID and not-COVID areas organized into specific emergency departments, medical wards, ICUs, and operating rooms (Table 1).

As part of the new procedure, healthcare workers do not move from one area to another. In non-COVID areas, patients and workers use surgical mask, gloves and gowns as personal protective equipment (PPE) as described by the World Health Organization. ${ }^{4}$

In COVID areas, FFP2 or FFP3 masks, eye protection, a double pair of gloves and a second gown are recommended during the aerosol-generating procedures (eg, swabbing, C-PAP therapy, etc). All masks must be certified according to the European standard (BS EN 149:2001). ${ }^{5}$

Author for correspondence: Prof Angelo Baggiani, E-mail: angelo.baggiani@med. unipi.it

Cite this article: Baggiani A, et al. (2020). Management of healthcare areas for the prevention of COVID-19 emergency in an Italian teaching hospital in Pisa, Tuscany: A hospital renovation plan. Infection Control \& Hospital Epidemiology, 41: 1368-1369, https://doi.org/10.1017/ice.2020.177
Table 1. Rational Division of Hospital in COVID and Non-COVID Areas

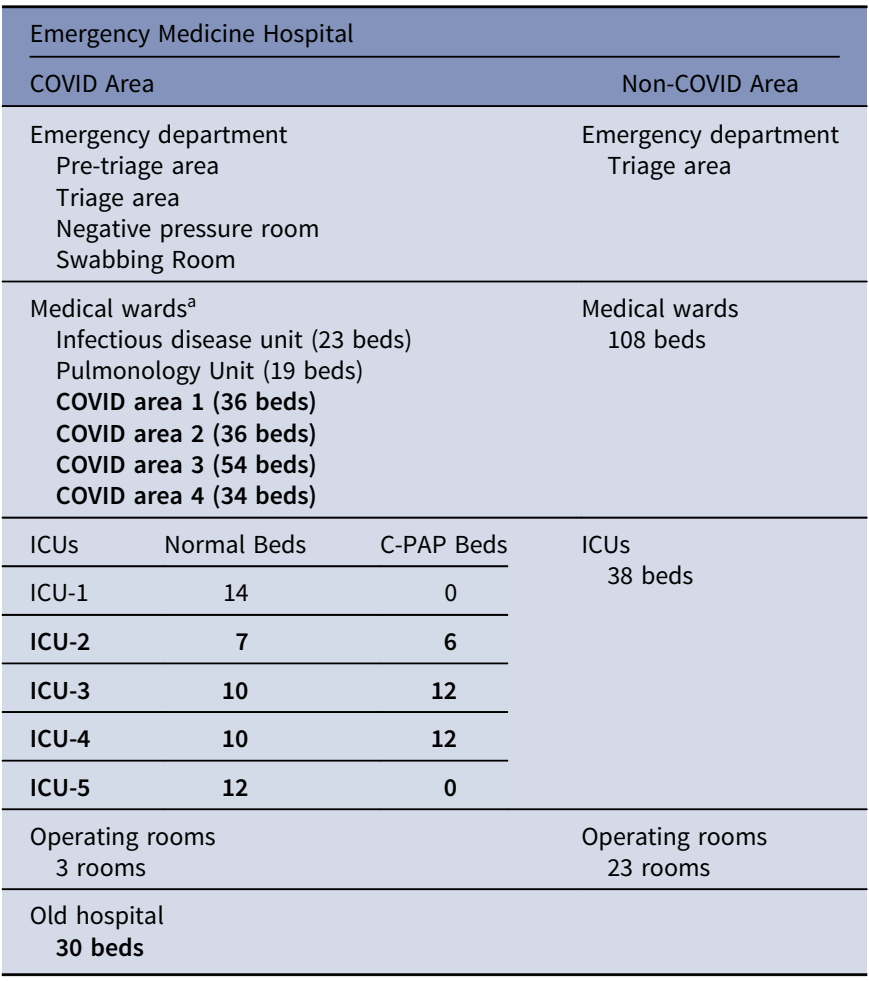

Note. ICU, intensive care unit; C-PAP, continuous positive airway pressure.

${ }^{a}$ New beds in medical wards and ICUs are shown in bold.

Considering the lack of sufficient beds in medicine wards and ICUs, the working group started a program to enlarge these areas. Beginning on March 19, several medical wards and operating rooms, located in different hospital units, have been repurposed to create new 160 COVID beds in medical wards (ie, COVID areas 1-4) and 69 COVID beds in ICUs (ie, COVID ICUs 2-5). In a old and previously disused hospital, a new area with 30 beds has been activated. Negative-pressure isolation room systems (-9 Pa) and high-efficiency particulate air (HEPA)-15 filters were installed in COVID ICUs to contain airborne microorganisms within the rooms. Negative-pressure isolation rooms are commonly used for COVID-19-positive patients with respiratory failure because 
they are usually treated with aerosol-generating C-PAP therapy (ie, they occupy C-PAP beds). ${ }^{6}$

On February 25, the hygiene and epidemiology unit and the prevention-protection service began providing telematic training to all hospital workers. Related course work has been developed to address various issues. A preliminary lesson was prepared on the epidemiological occurrences related to $\beta$-coronavirus outbreaks, with specific references to SARS-CoV-2. Furthermore, specific instruction on the importance of hospital disinfection, hand hygiene, and the use of PPE, has been provided to all healthcare personnel (ie, physicians, nurses, cleaner workers, etc). Finally, additional technical training has been provided to maintenance workers responsible for aeraulic systems to enhance their performance quality during this pandemic.

In conclusion, the emergence of the COVID-19 pandemic has required synergic cohesion of the working group to define the principal risks for patients and healthcare workers and to implement preventive measures such as PPE and training courses. At the same time, rapid renovation work in a hospital nearing completion was undertaken to enlarge the ICU areas for patients with respiratory failure. Overall, the structural division into non-COVID and COVID areas could be the best precautionary strategy to avoid the infectious risk between patients and staff.

Acknowledgments. None.

Financial support. No financial support was provided relevant to this article.
Conflicts of interest. All authors report no conflicts of interest relevant to this article.

\section{References}

1. Novel coronavirus disease 2019 (COVID-19) pandemic: increased transmission in the EU/EEA and the UK, sixth update. European Centre for Disease Prevention and Control website. https://www.ecdc.europa.eu/sites/default/ files/documents/RRA-sixth-update-Outbreak-of-novel-coronavirus-disease2019-COVID-19.pdf. Published March 12, 2020. Accessed April 21, 2020.

2. The Lancet Infectious Diseases Editorial. COVID-19, a pandemic or not? Lancet Infect Dis 2020;20:383.

3. Epidemia COVID-19. Aggiornamento nazionale [in Italian]. Istituto Superiore di Sanità website. https://www.epicentro.iss.it/coronavirus/bollettino/ Bollettino-sorveglianza-integrata-COVID-19_2-aprile-2020.pdf. Published April 2, 2020. Accessed April 21, 2020.

4. Rational use of personal protective equipment for coronavirus disease 2019 (COVID-19). World Health Organization website. https://apps.who.int/iris/ bitstream/handle/10665/331215/WHO-2019-nCov-IPCPPE_use-2020.1-eng. pdf. Published February 27, 2020. Accessed April 21, 2020.

5. Respiratory protective devices-filtering half masks to protect against particles. BS EN 149:2001. Requirements, testing, marking. British Standards website. http://www.nobelcert.com/DataFiles/FreeUpload/EN\%20149-2001\%20plus \%20A1-2009.pdf. Published July 2002. Accessed April 21, 2020.

6. Miller SL, Clements N, Elliott SA, Subhash SS, Eagan S, Radonovich LJ. Implementing a negative-pressure isolation ward for a surge in airborne infectious patients. Am J Infect Control 2017;45:652-659.

\title{
Risk factors for severe COVID-19 illness in healthcare workers: Too many unknowns
}

\author{
Pandora L. Wander MD, MS ${ }^{1,2}$ (]), Marika Orlov MD, $\mathrm{PhD}^{2}$, Susan E. Merel MD ${ }^{1}$ and Daniel A. Enquobahrie PhD, MPH, MD ${ }^{3}$ \\ ${ }^{1}$ Division of General Internal Medicine, Department of Medicine, University of Washington, Seattle, Washington, ${ }^{2}$ Department of Veterans Affairs, VA Puget Sound \\ Health Care System, Seattle, Washington and ${ }^{3}$ Department of Epidemiology, University of Washington, Seattle, Washington
}

To the Editor-We were very interested to read the recent letter by Zhou et al, "Protecting Chinese Healthcare Workers While Combating the 2019 Novel Coronavirus." 1 We agree that everything possible should be done to protect healthcare workers (HCWs) from developing COVID-19. We agree with the recommendations of Zhou et al including the importance of increasing production of personal protection equipment (PPE), training HCWs in proper use, and maintaining a high clinical suspicion for COVID-19 even in patients without respiratory symptoms. In addition, we call for more research into the risk factors leading to severe illness among HCWs, defined as COVID-19 requiring hospitalization or admission to the intensive care unit. ${ }^{2}$ Research in this area is sorely lacking, limiting implementation of evidence-based practices. Despite being younger and healthier than the general population with COVID-19, HCWs have similar rates of severe illness. In China, the proportion of HCWs with severe

Author for correspondence: Pandora L. Wander, E-mail: author@institution.edu Cite this article: Wander Pandora L., et al. (2020). Risk factors for severe COVID-19 illness in healthcare workers: Too many unknowns. Infection Control \& Hospital Epidemiology, 41: 1369-1370, https://doi.org/10.1017/ice.2020.178 illness decreased from $45 \%$ in early January 2020 to $9 \%$ after February 1,3 likely reflecting more consistent adoption of appropriate infection-control practices, including the use of PPE. However, severe illness among HCWs continues to be reported, suggesting that the use of currently approved infection control processes do not entirely prevent severe COVID-19 among HCWs. To explore risk factors for severe COVID-19 in HCWs, we performed structured searches using a Twitter analytics tool (Tweet Archivist, Seattle, WA) to identify news stories reported before March 16, 2020, about HCWs with severe COVID-19related illness ( $\mathrm{n}=6$ cases) (Table 1$)$. In most of these articles, neither high-risk host factors nor a clear high-inoculum exposure was evident, but in a few reports, potential exposure to inocula containing a high viral load was reported, including potential exposures to virus in stool.

In $10 \%$ of cases, gastrointestinal symptoms precede fever or respiratory symptoms by $1-2$ days. ${ }^{4}$ Furthermore, $60 \%$ of samples from the toilet, sink and door handles of an individual with SARS$\mathrm{CoV}-2$ were positive for viral RNA, even though the individual reported respiratory symptoms but not diarrhea. ${ }^{5}$ For HCWs, contact with surfaces and/or patients with these symptoms 